\title{
Robust Adaptive Beamforming against Signal Steering Vector Mismatch and Jammer Motion
}

\author{
Xiaojun Mao, ${ }^{1}$ Wenxing Li, ${ }^{1}$ Yingsong $\mathrm{Li}^{1}{ }^{1}$ Yaxiu Sun, ${ }^{1}$ and Zhuqun $\mathrm{Zhai}^{2}$ \\ ${ }^{1}$ College of Information and Communications Engineering, Harbin Engineering University, Harbin 150001, China \\ ${ }^{2}$ Institute of Systems, Beijing 100161, China
}

Correspondence should be addressed to Xiaojun Mao; wwwmaoxiaojun@126.com

Received 17 March 2015; Revised 8 June 2015; Accepted 16 June 2015

Academic Editor: Giuseppe Mazzarella

Copyright (C) 2015 Xiaojun Mao et al. This is an open access article distributed under the Creative Commons Attribution License, which permits unrestricted use, distribution, and reproduction in any medium, provided the original work is properly cited.

\begin{abstract}
Since adaptive beamformer suffers from output performance degradation in the presence of interference nonstationarity and signal steering vector mismatch, a novel robust null broadening adaptive beamforming is proposed. The proposed method is realized by the combination of projection transform and diagonal loading techniques. First, a new projection matrix with null broadening ability is constructed and then projects the array received data onto the projection matrix. With the diagonal loading technique, a new sample covariance matrix is obtained. The theoretical analysis shows that the projection transform operation can expand the incident direction of the interference and improve orthogonality between the signal-plus-interference and the noise subspaces; thus the proposed beamformer can effectively broaden the jammer null and enhance the null depth. The analytical expressions of the proposed algorithm are also provided, which are efficient and easily solved. Simulation results are presented and demonstrated that the proposed beamformer can provide strong robustness against signal steering vector mismatch and jammer motion.
\end{abstract}

\section{Introduction}

Adaptive beamforming can effectively suppress the interferences and noises by adaptively producing null at the direction of interference, and improve the array output signal to interference-plus-noise ratio (SINR), which have been widely used in radar, sonar, mobile communications, radio astronomy and other fields [1-3]. The common beamforming method such as minimum variance distortionless response (MVDR) beamformer is realized based on the premise that the array steering vector (ASV) of the desired signal is known accurately. In practical applications, the steering direction errors, wavefront distortion and coherent local scattering error, and so forth are unavoidable, and they cause ASV mismatches. The ASV mismatch will cause undesired target signal cancellation, resulting in serious performance degenerated $[4,5]$. Since the null at the direction of the interference is quite sharp, the MVDR beamformer is also known to severely degrade in the presence of nonstationary interferers. Such nonstationarity may be caused by the interferer motion or antenna array vibration, which may lead to mismatch between the training and application data of the adaptive weighting vector [6]. Moving interference is an especially serious problem in the antenna arrays. Therefore, adaptive beamforming techniques that can provide joint robustness against ASV mismatch and nonstationary interferer are required in these cases.

Null broadening allows the interferer to move in a certain area that is also contained within a single wide null, which can provide a simple and robust approach to null the moving interferers by producing broad trough at the direction of interference [7-12]. Mailloux and Zatman have proposed null broadening methods, respectively. Mailloux's method was realized by distributing a cluster of virtual equal-strength incoherent interferences around each original source [7]. Unlike Mailloux's method, Zatman utilized a certain bandwidth of the interference sources to replace the original narrowband interference, and the center frequency of the virtual interferences is the original narrowband interference [8]. Both of the two techniques are realized by the introduction of a conformal matrix "taper" into the original sample covariance matrix. After that, Guerci unified these 
two methods by introducing the concept of a "covariance matrix taper" (CMT) [9], which verified that the CMT operation reduced the array output power and resulted in null depth shallower. Another null broadening method is the derivative constraint $[10,11]$. Zatman studied the relationship between the CMT approach and derivative constraint in [12], which proved that the derivative constraint can be implemented as a CMT approach. In fact, these methods are identical, which have similar null broadening results, and they may not be sufficiently robust against ASV errors [13]. Recently, a new null broadening beamforming based on prior knowledge of the interference direction was proposed in [14], which can get a prespecified suppression level over a predefined sector by using a set of linear constraints (LCSS).

To improve the robustness of adaptive beamformer against ASV mismatch, many robust adaptive beamforming methods have been developed [15-22]. Diagonal loading is one of the commonly used methods [15-17], which can improve the robustness of array against ASV mismatch. However, it is very difficult to obtain the optimal diagonal loading factor. In [18], a robust Capon beamforming (RCB) algorithm has been proposed, which exploited the explicit uncertainty sets of the desired ASV. The RCB is a kind of diagonal loading techniques and its diagonal loading factor can be adjusted with the ellipsoidal uncertainty set. In addition, the robustness of the RCB has been greatly improved compared with the simplest diagonal loading method. It is worth noting that the performance of the RCB is mainly determined by the uncertain parameter set; however, the uncertainty in the ASV mismatch is also difficult to be known accurately in practice.

Another kind of robust adaptive beamforming based on steering vector estimation has been proposed in [19-21]. One of the famous methods was proposed by Hassanien to design a robust adaptive beamformer, which aims to estimate the difference between the actual and presumed ASVs and uses this difference to correct the erroneous presumed steering vector. In this beamforming, a quadratic convex optimization problem was obtained at each iteration to get the presumed steering vector. Unfortunately, it has high computational complexity and converges slowly. In another robust beamformer proposed by Khabbazibasmenj et al. in [20], the objective for estimating the ASV is the maximization of the beamformer output power, while the constraints are requirement that the estimated steering vector does not converge to any of the interference steering vectors or their linear combinations. The subspace projection robust beamforming (Eigenspace-RAB) has been proposed in [22, 23], which suffers from high probability of subspace swap and incorrect estimation of the signal-plus-interference subspace dimension at low SNR [20].

In many practical applications, the interference nonstationary and ASV mismatch may occur simultaneously. For the reasons mentioned above, the beamformer is required to widen the null and improve the robustness against ASV mismatch. Adaptive beamforming with joint robustness against ASV mismatch and interference nonstationarity has been proposed in [13], which is based on the idea of worst case performance optimization and covariance matrix mismatch. Although it can provide a certain joint robustness against
ASV mismatch and nonstationary interference, the method need know the uncertainty levels of ASV and array data matrix mismatch. In addition, it cannot get the analytical expression easily.

In this paper, a novel robust adaptive beamforming with null broadening is developed; the proposed method can provide joint robustness against ASV mismatch and interference nonstationarity. The proposed method is realized by the combination of projection transform and diagonal loading techniques. First, the steering vector correlation matrix in the angular sectors that the interferences may locate is constructed, and then we choose eigenvectors corresponding to the large eigenvalues of the correlation matrix to establish a new projection matrix and project the array received data onto the projection matrix. Finally, with the diagonal loading technique, a new covariance matrix can be obtained. The proposed method, which can be solved directly by using analytical expression without iterative solution, can effectively broaden jammer null and enhance null depth and hence can provide a good performance to suppress strong fast-moving interferers. Moreover, it also provides strong robustness against ASV mismatch. Simulation results show that the proposed beamformer possesses a superior performance compared to existing popular adaptive beamformers. Thus, the proposed method is promising in practical applications.

\section{The Signal Model}

We consider the narrowband signals impinge upon an $N$ dimension omnidirectional array in the far field. The incident angle of desired signal is $\theta_{0}$. $P$ independent interferences from different directions are $\hat{\theta}_{p}, p=1,2, \ldots, P$. Thus, the received data $\mathbf{X}(i)$ can be expressed as

$$
\mathbf{X}(i)=\mathbf{A S}(i)+\mathbf{N}(i)
$$

where $\mathbf{X}(i)=\left[x_{1}(i), x_{2}(i), \ldots, x_{N}(i)\right]^{T}$ is an $N \times 1$ snap data vector. $i$ is the time index, and $\mathbf{S}(i)=\left[s_{0}(i), s_{2}(i), \ldots, s_{m}(i)\right.$, $\left.\ldots, s_{P}(i)\right]^{T}$ is an envelope vector of the signal and interferences, where $s_{m}(i)$ is complex waveform of the $m$ th signal. $\mathbf{N}(i)=\left[n_{1}(i), n_{2}(i), \ldots, n_{N}(i)\right]^{T}$ is a noise vector with zero mean and variance of $\sigma_{n}^{2}$. $\mathbf{A}=\left[\mathbf{a}\left(\theta_{0}\right), \mathbf{a}\left(\hat{\theta}_{2}\right), \ldots, \mathbf{a}\left(\hat{\theta}_{P}\right)\right]$ is a matrix with columns of the steering vectors, where $\mathbf{a}\left(\theta_{p}\right)=$ $\left[1, e^{j k \sin \left(\widehat{\theta}_{p}\right)}, \ldots, e^{j(N-1) k \sin \left(\widehat{\theta}_{p}\right)}\right]^{T}, p=0,2 \ldots, P$, in the $\hat{\theta}_{p}$ direction, and $k=(2 \pi / \lambda) d$.

Assuming that the signal, interferences, and noise are statistically independent, the data covariance can be written as

$$
\mathbf{R}=E\left\{\mathbf{X}(i) \mathbf{X}^{H}(i)\right\}=\mathbf{A R}_{s} \mathbf{A}^{H}+\sigma_{n}^{2} \mathbf{I}
$$

where $E\{\}$ denotes an expectation operator; $\mathbf{R}_{s}=E\left\{s(i) s^{H}(i)\right\}$ represents the autocorrelation matrix of signal-plusinterference, I is the unit matrix, and ()$^{H}$ denotes the conjugate transposition. 
The standard MVDR beamformer, which is a linearly constrained quadratic optimization problem, can be implemented as follows:

$$
\begin{array}{cl}
\min _{\mathbf{w}} & \mathbf{w}^{H} \mathbf{R}_{i+n} \mathbf{w} \\
\text { subject to } & \mathbf{w}^{H} \mathbf{a}\left(\theta_{0}\right)=1,
\end{array}
$$

where $\mathbf{R}_{i+n}$ is the covariance matrix of inference-plus-noise. In practice, this matrix is commonly replaced by the sample covariance matrix with $K$ snapshots and is rewritten as $\widehat{\mathbf{R}}=$ $(1 / K) \sum_{i=1}^{K} \mathbf{X}(i) \mathbf{X}^{H}(i)$. Then, the optimal solution of (3) is

$$
\mathbf{w}=\frac{\widehat{\mathbf{R}}^{-1} \mathbf{a}\left(\theta_{0}\right)}{\left(\mathbf{a}^{H}\left(\theta_{0}\right) \widehat{\mathbf{R}}^{-1} \mathbf{a}\left(\theta_{0}\right)\right)} .
$$

The above solution is commonly regarded as the sample matrix inverse (SMI). The SMI beamforming produces sharp null in the direction of interference and provides good interference suppression performance when the ASV is known accurately. In practice, array imperfections including, for example, steering direction errors, wavefront distortion, and coherent local scattering are unavoidable, and they cause ASV mismatches. As a result, the SMI beamforming may suppress the desired signal, which will deteriorate the performance of the beamformer. The SMI beamforming is also unable to provide high performance when the adaptive weights are not sufficiently fast to nonstationary [13].

\section{The Proposed Beamformer}

In this paper, we proposed a novel robust null broadening adaptive beamforming. The proposed method can effectively broaden the jammer null and enhance the null depth, which can suppress the strong fast-moving interference and provide high output SINR. In this section, we give the formulation of the new projection matrix and then present detailed theoretical analysis and geometrical interpretation.

3.1. Null Broadening Based on Projection Transform. First, we use the Capon spatial spectrum estimator to estimate the interference directions [24]. Then, the power spectrum in the entire spatial domain can be constructed as

$$
\widehat{P}(\theta)=\frac{1}{\mathbf{a}^{H}(\theta) \widehat{\mathbf{R}}^{-1} \mathbf{a}(\theta)} .
$$

Assuming that $\Theta$ is the angular sector in which the desired signal is located and $\bar{\Theta}$ is the complement sector of $\Theta$, we search for the spectral peaks of $\widehat{P}(\theta)$ in $\bar{\Theta}$, and the location of these peaks should correspond to the interferers. The set of all the angles corresponding to these spectral peaks is $\Gamma=$ $\left\{\theta_{p}, p=1,2, \ldots, P\right\}$. It is worth noting that the estimated interference directions $\theta_{p}$ may be not very accurate. Thus, the following analysis is given to show that the broadening null can offset the error, and the interference can still be effectively suppressed as the simulation results demonstrated.

We assume the angle mismatch of interference direction is $\Delta \theta$ at some point; since the interference is nonstationary,
$\Delta \theta$ is uniformly obtained from the interval $[-\delta / 2, \delta / 2]$. Then, the probability density function of $\Delta \theta$ can be expressed as

$$
f(\Delta \theta)= \begin{cases}\frac{1}{\delta} & -\frac{\delta}{2}<\Delta \theta<\frac{\delta}{2} \\ 0 & \text { else, }\end{cases}
$$

where $\delta$ determines the null width.

The actual interference direction $\widehat{\theta}_{p}$ is modified as

$$
\widehat{\theta}_{p}=\theta_{p}+\Delta \theta \text {. }
$$

It is easy to see that $\sin \left(\widehat{\theta}_{p}\right)$ is approximate uniform distribution in the interval $\left[\sin \left(\theta_{p}\right)-(\delta / 2) \cos \left(\theta_{p}\right), \sin \left(\theta_{p}\right)+\right.$ $\left.(\delta / 2) \cos \left(\theta_{p}\right)\right]$. Denote $u_{p}=\sin \left(\theta_{p}\right)$, and define the correlation matrix of the steering vector:

$$
\mathbf{R}_{\theta}=\sum_{p=1}^{P} \int_{\sin \theta_{p}-(\delta / 2) \cos \theta_{p}}^{\sin \theta_{p}+(\delta / 2) \cos \theta_{p}} f\left(u_{p}\right) \mathbf{a}\left(\theta_{p}\right) \mathbf{a}^{H}\left(\theta_{p}\right) d_{u_{p}},
$$

where $f\left(u_{p}\right)$ is the probability density function of $u_{p}$, and then $(8)$ can be rewritten as

$$
\mathbf{R}_{\theta}=\sum_{p=1}^{P} \int_{\sin \theta_{p}-(\delta / 2) \cos \theta_{p}}^{\sin \theta_{p}+(\delta / 2) \cos \theta_{p}} \frac{1}{\delta \cos \theta_{p}} \mathbf{a}\left(\theta_{p}\right) \mathbf{a}^{H}\left(\theta_{p}\right) d_{u_{p}} .
$$

The $(m, n)$ th $(1 \leq m, n \leq N)$ element of matrix $\mathbf{R}_{\theta}$ can be expressed as

$$
\begin{aligned}
\mathbf{R}_{\theta m n} & =\sum_{p=1}^{P} \int_{\sin \theta_{p}-(\delta / 2) \cos \theta_{p}}^{\sin \theta_{p}+(\delta / 2) \cos \theta_{p}} \frac{1}{\delta \cos \theta_{p}} e^{j(m-n) k u_{p}} d_{u_{p}} \\
& =\sum_{p=1}^{P} \frac{\sin \left((m-n) k \delta \cos \theta_{p}\right)}{(m-n) k \delta \cos \theta_{p}} e^{j(m-n) k \sin \theta_{p}} \\
& =\sum_{p=1}^{P} \mathbf{a}\left(\theta_{p}\right) \mathbf{a}^{H}\left(\theta_{p}\right) \mathbf{C}\left(\theta_{p}\right)_{m n},
\end{aligned}
$$

where $\mathbf{C}\left(\theta_{p}\right)_{m n}=\sin \left((m-n) k \delta \cos \theta_{p}\right) /(m-n) k \delta \cos \theta_{p}$. Since $\theta_{p}$ is constant, $\mathbf{C}\left(\theta_{p}\right)_{m n}$ can be replaced by $\mathbf{C}_{m n}$ :

$$
\mathbf{C}_{m n}=\frac{\sin ((m-n) k \delta)}{(m-n) k \delta} .
$$

Then, (8) can be expressed as

$$
\mathbf{R}_{\theta}=\sum_{p=1}^{P} \mathbf{Z}_{p} \circ \mathbf{C}
$$

where ( $\circ$ ) denotes the Schur-Hadamard matrix product and $\mathbf{Z}_{p}=\mathbf{a}\left(\theta_{p}\right) \mathbf{a}^{H}\left(\theta_{p}\right)$. The correlation matrix $\mathbf{R}_{\theta}$ can be eigendecomposed as

$$
\mathbf{R}_{\theta}=\sum_{n=1}^{N} \mu_{\theta_{n}} \overline{\boldsymbol{v}}_{\theta_{n}} \overline{\boldsymbol{v}}_{\theta_{n}}^{H}, \quad \mu_{\theta_{1}} \geq \mu_{\theta_{2}} \geq \cdots \geq \mu_{\theta_{N}},
$$


where $\mu_{\theta_{n}}(1 \leq n \leq N)$ are the eigenvalues of $\mathbf{R}_{\theta}$ in descending and $\overline{\boldsymbol{v}}_{\theta_{n}}$ are the corresponding eigenvectors. In all the eigenvalues, we can extract $M$ large eigenvalues, which can be expressed as

$$
\frac{\sum_{n=1}^{M} \mu_{\theta_{n}}}{\sum_{n=1}^{N} \mu_{\theta_{n}}} \geq \xi
$$

where $\xi$ is the constraint parameter that determines the selection of the number of the base vectors. The $M$ eigenvectors are the base vectors, which were used to establish an interference subspace. The interference subspace can be expressed as

$$
\bar{H}=\operatorname{span}\left\{\overline{\boldsymbol{v}}_{\theta_{1}}, \overline{\boldsymbol{v}}_{\theta_{2}}, \ldots, \overline{\boldsymbol{v}}_{\theta_{M}}\right\} .
$$

Define the projection matrix $\mathbf{T}$ as

$$
\mathbf{T}=\sum_{n=1}^{M} \overline{\boldsymbol{v}}_{\theta_{n}} \overline{\boldsymbol{v}}_{\theta_{n}}^{H} .
$$

The idempotent projection matrix of $\mathbf{T}$ can be easily observed. Projecting $\mathbf{X}(i)$ onto $\mathbf{T}$, we get

$$
\widetilde{\overline{\mathbf{X}}}(i)=\mathbf{T X}(i) \text {. }
$$

According to (2), the covariance matrix of the array can be modified as

$$
\widehat{\overline{\mathbf{R}}}=\frac{1}{K} \sum_{i=1}^{K} \widetilde{\overline{\mathbf{X}}}(i) \widetilde{\overline{\mathbf{X}}}^{H}(i)=\mathbf{T} \widehat{\mathbf{R}} \mathbf{T}^{H}
$$

Since the projection matrix $\mathbf{T}$ is a unitary matrix, this means

$$
\mathbf{T} \mathbf{T}^{H}=\mathbf{I} .
$$

Thus, (18) is rewritten as

$$
\widehat{\overline{\mathbf{R}}}=\mathbf{T} \mathbf{A} \widehat{\mathbf{R}}_{s} \mathbf{A}^{H} \mathbf{T}^{H}+\sigma_{n}^{2} \mathbf{I} .
$$

From the discussions above, we know that the projection matrix $\mathbf{T}$ is obtained from $\mathbf{R}_{\theta}$, and hence the directions information of the interferences has already been involved in $\mathbf{T}$; that means that the projection matrix $\mathbf{T}$ can play a role in expanding the beam null.

3.2. Theoretical Analysis. According to Schmidt's orthogonal subspace resolution theory, $\widehat{\mathbf{R}}$ can be decomposed as [25]

$$
\begin{aligned}
& \widehat{\mathbf{R}}=\overline{\mathbf{U}} \bar{\Sigma} \overline{\mathbf{U}}^{H}=\left[\begin{array}{ll}
\overline{\mathbf{U}}_{S} & \overline{\mathbf{U}}_{N}
\end{array}\right] \bar{\Sigma}\left[\begin{array}{ll}
\overline{\mathbf{U}}_{S} & \overline{\mathbf{U}}_{N}
\end{array}\right]^{H} \\
& =\overline{\mathbf{U}}_{S} \bar{\Sigma}_{S} \overline{\mathbf{U}}_{S}^{H}+\overline{\mathbf{U}}_{N} \bar{\Sigma}_{N} \overline{\mathbf{U}}_{N}^{H} \\
& =\sum_{i=1}^{P+1} \bar{\mu}_{i} \overline{\boldsymbol{v}}_{i} \overline{\boldsymbol{v}}_{i}^{H}+\sum_{j=P+2}^{N} \bar{\mu}_{j} \overline{\boldsymbol{v}}_{j} \overline{\boldsymbol{v}}_{j}^{H},
\end{aligned}
$$

where $\overline{\mathbf{U}}$ is an eigenvector of covariance matrix $\widehat{\mathbf{R}} \cdot \bar{\Sigma}$ is a diagonal matrix, which is obtained from corresponding eigenvalues. $\overline{\mathbf{U}}_{S}=\left[\overline{\boldsymbol{v}}_{1}, \overline{\boldsymbol{v}}_{2}, \ldots, \overline{\boldsymbol{v}}_{P+1}\right]$ represents the signalplus-interference subspace, which is formed by the $P$ interferences and one desired signal; $\overline{\mathbf{U}}_{N}=\left[\overline{\boldsymbol{v}}_{P+2}, \overline{\boldsymbol{v}}_{P+3}, \ldots, \overline{\boldsymbol{v}}_{N}\right]$ represents the noise subspace; $\Sigma_{S}=\operatorname{diag}\left\{\mu_{1}, \mu_{2}, \ldots, \mu_{P+1}\right\}$ is the eigenvalues of the desired signal and interferences; and $\Sigma_{N}=\operatorname{diag}\left\{\mu_{P+2}, \mu_{P+3}, \ldots, \mu_{N}\right\}$ is the eigenvalues of noise.

In practical application, the number of snapshots, the environment of signal, and the perturbations of array may cause the orthogonality deterioration, resulting in the fact that the desired result cannot be achieved. In the proposed method, the project transformation can not only broaden the nulls but also improve the orthogonality between the signal and noise subspaces. The sample covariance matrix $\widehat{\overline{\mathbf{R}}}$ can be rewritten as

$$
\begin{aligned}
& \widehat{\widehat{\mathbf{R}}}=\mathbf{T} \overline{\mathbf{U}} \bar{\Sigma} \overline{\mathbf{U}}^{H} \mathbf{T}^{H}=\mathbf{T}\left[\begin{array}{ll}
\overline{\mathbf{U}}_{S} & \overline{\mathbf{U}}_{N}
\end{array}\right] \bar{\Sigma}\left[\begin{array}{ll}
\overline{\mathbf{U}}_{S} & \overline{\mathbf{U}}_{N}
\end{array}\right]^{H} \mathbf{T}^{H} \\
& =\mathbf{T} \overline{\mathbf{U}}_{S} \bar{\Sigma}_{S} \overline{\mathbf{U}}_{S}^{H} \mathbf{T}^{H}+\mathbf{T} \overline{\mathbf{U}}_{N} \bar{\Sigma}_{N} \overline{\mathbf{U}}_{N}^{H} \mathbf{T}^{H} .
\end{aligned}
$$

From (8) to (12), we can see that the base vectors are located in interference subspace. Therefore, the subspace $\bar{H}$ which is spanned by the base vectors should also be orthogonal to $\overline{\mathbf{U}}_{N}$, which means $\left\{\overline{\boldsymbol{v}}_{\theta_{1}}, \overline{\boldsymbol{v}}_{\theta_{2}}, \ldots, \overline{\boldsymbol{v}}_{\theta_{M}}\right\} \perp \overline{\mathbf{U}}_{N}$. In practice application, the projection matrix can strengthen the signal-plus-interference subspace and suppress the noise subspace. Thus, the orthogonality between the signal-plusinterference and noise subspace is improved. As we all know, if the spread of noise eigenvalues is minimized, the effects of randomly shaped noise eigenbeaming might be reduced and the adapted beam approaches the ideal pattern [15].

3.3. Geometric Explanation. We can investigate the projection transform from the view of geometry analysis. For the practical applications, the number of snapshots may cause errors to the covariance matrix eigenvectors of the actual signal, which is denoted as

$$
\overline{\boldsymbol{v}}_{i}=\boldsymbol{v}_{i}+\Delta \overline{\boldsymbol{v}}_{i}
$$

where $\boldsymbol{v}_{i}$ is the ideal eigenvector and $\Delta \overline{\boldsymbol{v}}_{i}$ denotes an unknown mismatch. Projecting the array received data onto the projection matrix is equivalent to projecting the eigenvectors to the projection matrix and then we have

$$
\mathbf{T} \overline{\boldsymbol{v}}_{i}=\mathbf{T}\left(\boldsymbol{v}_{i}+\Delta \overline{\boldsymbol{v}}_{i}\right)=\mathbf{T} \boldsymbol{v}_{i}+\mathbf{T} \Delta \overline{\boldsymbol{v}}_{i}
$$

Since $\mathbf{T}$ is idempotent and lies in the same subspace as the ideal eigenvector, thus

$$
\mathrm{T} v_{i}=v_{i}
$$

Substituting (25) to (24), (24) can be rewritten as

$$
\mathbf{T} \overline{\boldsymbol{v}}_{i}=\mathbf{T}\left(\boldsymbol{v}_{i}+\Delta \overline{\boldsymbol{v}}_{i}\right)=\mathbf{T} \boldsymbol{v}_{i}+\mathbf{T} \Delta \overline{\boldsymbol{v}}_{i}=\boldsymbol{v}_{i}+\mathbf{T} \Delta \overline{\boldsymbol{v}}_{i}
$$

It can be seen that $\mathbf{T} \Delta \overline{\boldsymbol{v}}_{i}$ is located in projection matrix space, and hence the error is smaller. Therefore, the eigenvectors are closer to the ideal case, which can be illustrated in Figure 1. 


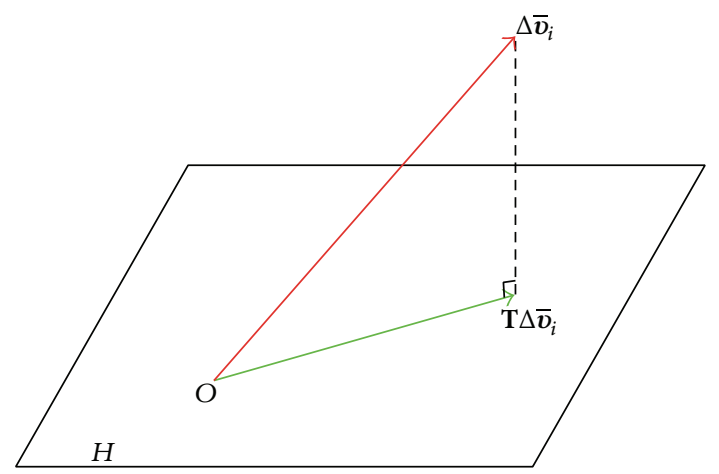

FIgURE 1: Geometrical interpretation on the relationship between the subspace $\bar{H}$, the mismatch $\Delta \overline{\boldsymbol{v}}_{i}$, and $\mathbf{T} \Delta \overline{\boldsymbol{v}}_{i}$.

As can be seen from Figure $1, \Delta \overline{\boldsymbol{v}}_{i}$ is reduced, and

$$
\left\|\mathbf{T} \Delta \overline{\boldsymbol{v}}_{i}\right\|_{2} \leq\left\|\Delta \overline{\boldsymbol{v}}_{i}\right\|_{2},
$$

where $\|\cdot\|_{2}$ denotes the Euclidean norm. Since the error is reduced, the disturbance of noise eigenvalues becomes small, resulting in the orthogonality between the signal-plusinterference and noise subspaces close to the ideal case, which is consistent with the theoretical analysis.

From the above analysis, we can see that the projection matrix contains the directions information of the interferences, which can broaden the jammer null. In addition, the signal-plus-interference subspace is strengthened, which can improve the orthogonality between the signal-plusinterference and noise subspaces, making the jammer null deeper and the inhibition gains increased.

3.4. Diagonal Loading. Diagonal loading is widely used to improve the robustness of the beamformers [15-17]. In this paper, a small positive number is added to the diagonal of the covariance matrix $\widehat{\overline{\mathbf{R}}}$ and is given by

$$
\widetilde{\overline{\mathbf{R}}}=\widehat{\overline{\mathbf{R}}}+\lambda \mathbf{I}=\mathbf{T} \widehat{\mathbf{R}} \mathbf{T}^{H}+\lambda \mathbf{I},
$$

where $\lambda$ is the diagonal loading level.

Up to now, the new sample covariance matrix $\widetilde{\overline{\mathbf{R}}}$ is obtained. By using $\widetilde{\overline{\mathbf{R}}}$, we can get the adaptive weight vector:

$$
\begin{aligned}
\widetilde{\mathbf{W}} & =\frac{\widetilde{\mathbf{R}}^{-1} \mathbf{a}\left(\theta_{0}\right)}{\mathbf{a}^{H}\left(\theta_{0}\right) \widetilde{\overline{\mathbf{R}}}^{-1} \mathbf{a}\left(\theta_{0}\right)} \\
& =\frac{\left(\mathbf{T} \widehat{\mathbf{R}} \mathbf{T}^{H}+\lambda \mathbf{I}\right)^{-1} \mathbf{a}\left(\theta_{0}\right)}{\mathbf{a}^{H}\left(\theta_{0}\right)\left(\mathbf{T} \widehat{\mathbf{R}} \mathbf{T}^{H}+\lambda \mathbf{I}\right)^{-1} \mathbf{a}\left(\theta_{0}\right)} .
\end{aligned}
$$

Generally, each step of the proposed beamforming can be described in detail as follows.

Step 1. Calculate the covariance matrix $\widehat{\mathbf{R}}=(1 / K) \sum_{i=1}^{K} \mathbf{X}(i)$ $\mathbf{X}^{H}(i)$.
Step 2. Calculate the spatial power spectrum $\widehat{P}(\theta)$ with (5); the coarse directions of the interferences $\theta_{p}, p=1,2, \ldots, P$, can be estimated by searching the peaks of $\widehat{P}(\theta)$ in $\bar{\Theta}$.

Step 3. Calculate the steering vectors autocorrelation matrix of the interferences $\mathbf{Z}=\sum_{p=1}^{P} \mathbf{a}\left(\theta_{p}\right) \mathbf{a}^{H}\left(\theta_{p}\right)$; then $\mathbf{R}_{\theta}$ can be obtained from (12).

Step 4. Let $\mathbf{R}_{\theta}=\sum_{n=1}^{N} \mu_{\theta_{n}} \overline{\boldsymbol{v}}_{\theta_{n}} \overline{\boldsymbol{v}}_{\theta_{n}}^{H}, \mu_{\theta_{1}} \geq \mu_{\theta_{2}} \geq \cdots \geq \mu_{\theta_{N}}$ by using eigendecomposition; calculate the projection matrix $\mathbf{T}=\sum_{n=1}^{M} \overline{\boldsymbol{v}}_{\theta_{n}} \overline{\boldsymbol{v}}_{\theta_{n}}^{H}$.

Step 5. Project the array covariance matrix onto $\mathbf{T}$ :

$$
\widehat{\overline{\mathbf{R}}}=\mathbf{T} \widehat{\mathbf{R}} \mathbf{T}^{H} .
$$

Step 6. Utilize the diagonal loading in (28):

$$
\widetilde{\overline{\mathbf{R}}}=\widehat{\overline{\mathbf{R}}}+\lambda \mathbf{I}=\mathbf{T} \widehat{\mathbf{R}} \mathbf{T}^{H}+\lambda \mathbf{I} .
$$

Step 7. Compute the adaptive weight vector $\widetilde{\mathbf{W}}$ with (29).

It can be seen from (29) that the adaptive weight vector of the proposed method can be obtained by solving the analytical expression without iterative solution.

In our algorithm, forming the matrix product $\mathbf{Z}$ in Step 2 and T in Step 4 each has a complexity of $O\left(N^{2}\right)$. The main complexity is the eigendecomposition operation on the matrix $\mathbf{R}_{\theta}$ and matrix inverse operation on $\widetilde{\overline{\mathbf{R}}}$, of which each has a complexity of $O\left(N^{3}\right)$ [18]. Therefore, the overall complexity of our beamformer is $O\left(2 N^{3}+2 N^{2}\right)$. The computational complexity of the simplest SMI beamformer is $O\left(N^{3}\right)$. The complexity for RCB and Eigenspace-based beamforming is $O\left(N^{3}\right)$ [18]. The beamformer in [13] has a complexity of $O\left(\rho N^{3}\right)$, where $\rho$ is the number of iterations. The SQP method in [19] has a complexity of $O\left(N^{3.5}\right)$, and the beamforming method in [20] has a complexity of $O\left(N^{6}\right)$. The computational complexity of the proposed method is smaller than the beamformers in $[13,19]$ and SQP method. Though the complexity of our method is higher than that of SMI and $\mathrm{RCB}$, our proposed method can significantly enhance robustness performance.

\section{Simulation Results}

We consider a uniform linear array of $N=10$ elements spaced a half-wavelength distance. Additive noise is modeled as independent complex Gaussian noise with zero mean and unit variance. The interference-to-noise rations (INRs) of the interferences are both $30 \mathrm{~dB}$. The desired signal incidents are from $0^{\circ}$. In all simulations, 100 Monte Carlo runs are used to obtain each simulated point.

Simulation Example 1 (null broadening comparisons). In this simulation, we set the number of the base vectors $M=6$ and the width of the null $\delta=10^{\circ}$ and the diagonal loading factor $\lambda=0.0100$, and the ASV is exactly known. The number of snapshots is $K=200$. The input SNR is $0 \mathrm{~dB}$. 


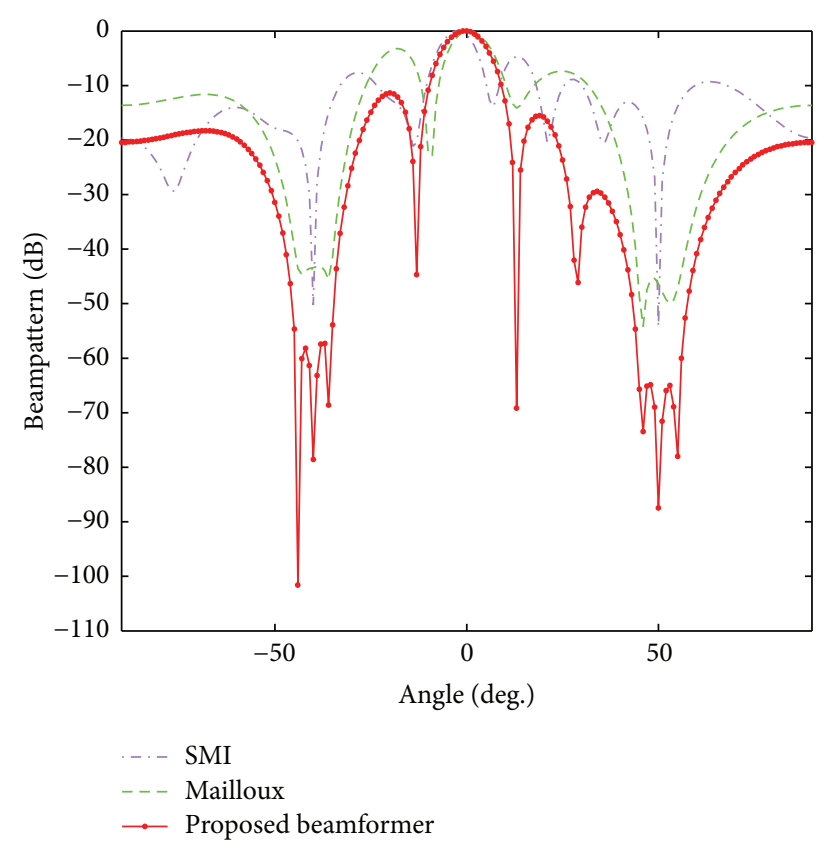

FIGURE 2: Normalized null broadened beampattern plots.

Two independent interferences are from the directions $-40^{\circ}$ and $50^{\circ}$. Here, the performance of the proposed algorithm is compared with Mailloux's approach [7].

The normalized beampatterns of these beamformers are shown in Figure 2. It can be seen that the SMI beamforming produces narrow and deep nulls in the directions of interferences and the null depth is about $-50 \mathrm{~dB}$. Mailloux's method can produce wide jammer nulls; however, the null depth is only $-45 \mathrm{~dB}$. Therefore, the depth of Mailloux's nulls may not be sufficient to suppress strong jamming. In addition, Mailloux's method has large side lobe level. The proposed algorithm produced angular widening for the jammer nulls in the adapted pattern. It can be seen that the proposed method exhibits deeper nulls compared to Mailloux's method. The null depth reaches about $-70 \mathrm{~dB}$ which provides better performance for interference suppression. Moreover, the side lobe level is also depressed.

Simulation Example 2 (performance of fast-moving interference suppression). In this simulation, we assume a moving interference is initially located in $-45^{\circ}$ direction and moves with $v_{\theta}$ per snapshot. We set $v_{\theta}=0.01^{\circ}$ and $v_{\theta}=0.05^{\circ}$, respectively, which means the interference is moving with the time-varying directions $\theta(k)=-45^{\circ}+0.01^{\circ} i$ and $\theta(k)=-45^{\circ}+0.05^{\circ} i$, respectively. The number of snapshots is $K=150$. Other simulation parameters are the same as Simulation Example 1. The proposed beamforming algorithm is investigated and compared with the diagonally loaded SMI (LSMI) [15], LCSS [14]. The diagonal loading factor for LSMI and DL-Mailloux is selected as twice as the noise power. As for LCSS method, the diagonal loading factor to noise power ration is $10 \mathrm{~dB}$ as suggested in [14].

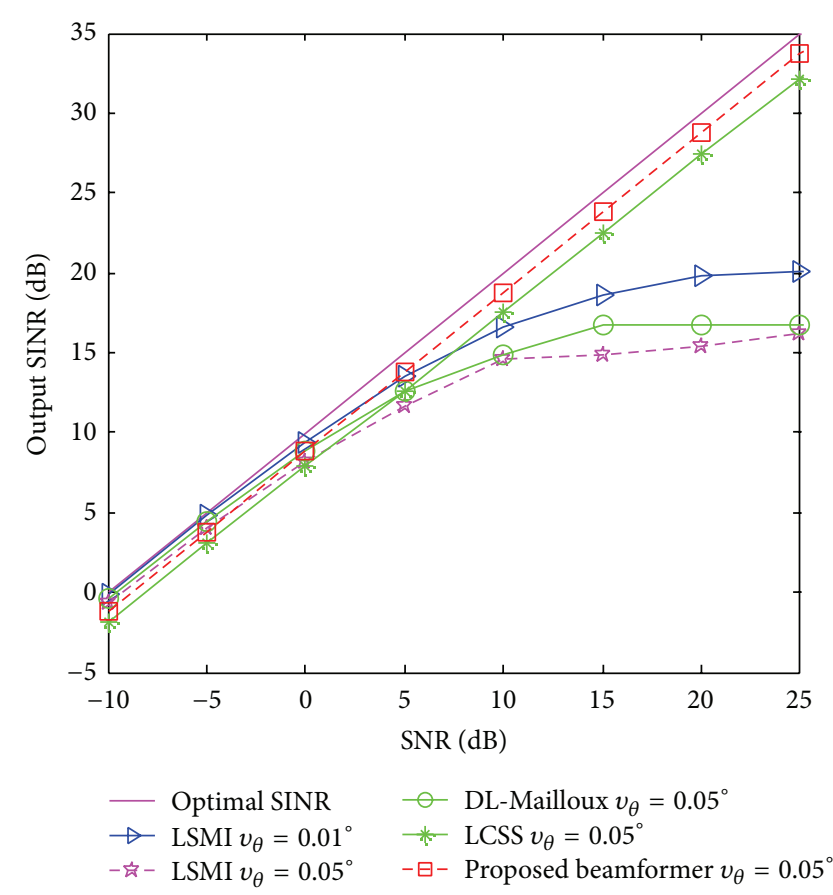

FIgURE 3: Output SINR of beamformers versus input SNR.

Figure 3 compares the output SINR of the beamformers versus SNR. We can see that the LSMI suffers performance degraded with the increase of $v_{\theta}$. The performance of DLMailloux method is better than LSMI, which can provide certain robustness against moving interference. The proposed method has the best performance among all the tested methods. The output SINR is better than LCSS, which is closer to the optimal one.

Simulation Example 3 (desired ASV mismatch due to wavefront distortion). In this simulation, we consider that the desired signal ASV is distorted by the effects of wave propagation in an inhomogeneous medium which is used in [20]. In particular, independent-increment phase distortions are accumulated from the components of the presumed ASV. Assuming that the phase increments are fixed in each simulation run and are independently chosen from a Gaussian random generator with zero mean and standard deviation 0.04 , we set the number of the base vectors $M=6$ and the width of the null $\delta=10^{\circ}$ and the diagonal loading factor $\lambda=0.0100$, and the input SNR is $0 \mathrm{~dB}$. The proposed beamforming algorithm is investigated and compared with the diagonally loaded SMI (LSMI) [15], RCB [18], Eigenspacebased beamformer [22], the sequential programming- (SQP-) based beamformer [19], and the beamformer of [20]. The optimal parameter $\varepsilon=0.3 \mathrm{~N}$ is used for the $\mathrm{RCB}$, while the diagonal loading factor of LSMI is selected as twice as the noise power.

To make the figure clear, only the normalized beampatterns of SMI, RCB, and the proposed beamformer are given in Figure 4. It can be seen from Figure 4 that the SMI completely fails in a wavefront distortion environment, which produces 


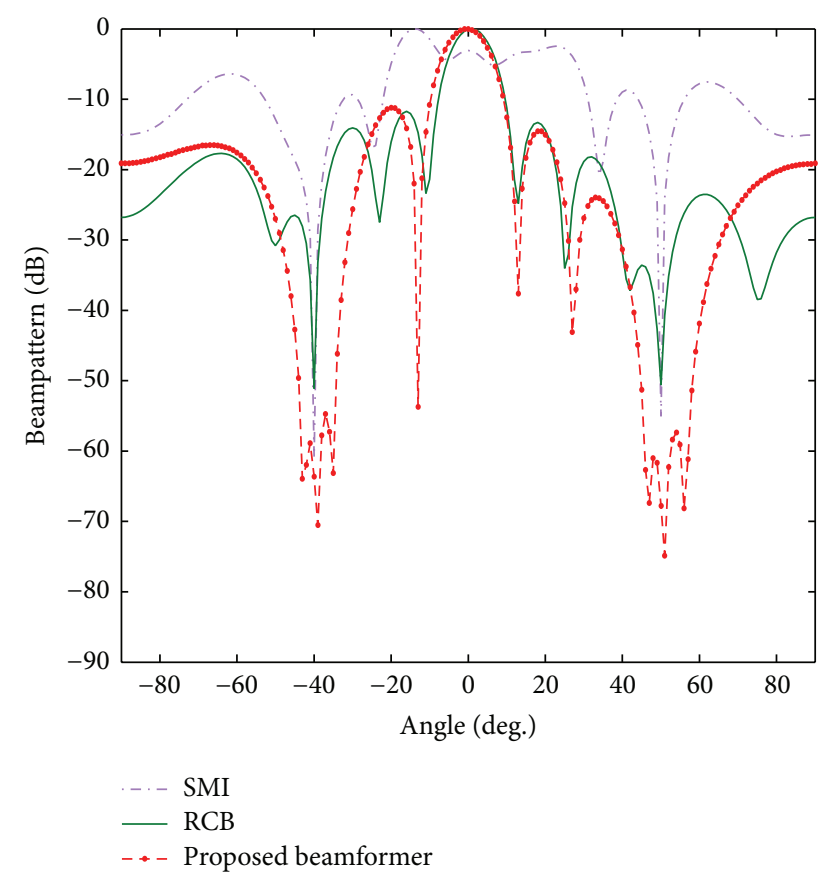

FIGURE 4: Normalized beampattern plots in absence of ASV mismatch due to wavefront distortion.

a null in the direction of the desired signal. The performance of the RCB is better than the SMI. However, its interference rejection level is degraded since the null may not be deep enough. The proposed method shows excellent performance in interference suppressions, providing deep and wide nulls in the directions of inferences, and its response peaks at the actual direction of desired signal.

Figure 5 shows the output SINR of the beamformers versus input SNR. It can be seen in Figure 5 that the output SINR of SMI decreased sharply with an increment of the input SNR. The Eigenspace-based beamforming suffers severe performance degradation due to the inaccurate estimate of the subspace at low SNR. The proposed algorithm has the best performance compared to other beamformers in wavefront distortion environment. It is found that our beamformer outperforms the Eigenspace-based beamformer, RCB, and $S Q P$ at high SNR values, which is very close to the optimal one.

Simulation Example 4 (desired ASV mismatch due to coherent local scattering). In this simulation, we consider that the desired ASV is distorted by local scattering effects. The actual ASV is formed by several signal paths, which can be modeled as

$$
\mathbf{a}=\mathbf{a}_{0}+\sum_{i=1}^{T} \exp \left(j \varphi_{i}\right) \mathbf{a}\left(\theta_{i}\right),
$$

where $\mathbf{a}_{0}$ denotes the direct path and $T$ represent the number of coherently scattered paths. The $i$ th path $\mathbf{a}\left(\theta_{i}\right)$ is modeled as a plane wave which is incident on the array at the direction of $\theta_{i}$. The parameter $\varphi_{i}$ represents the path phases that are independently and uniformly drawn from the interval $[0,2 \pi]$

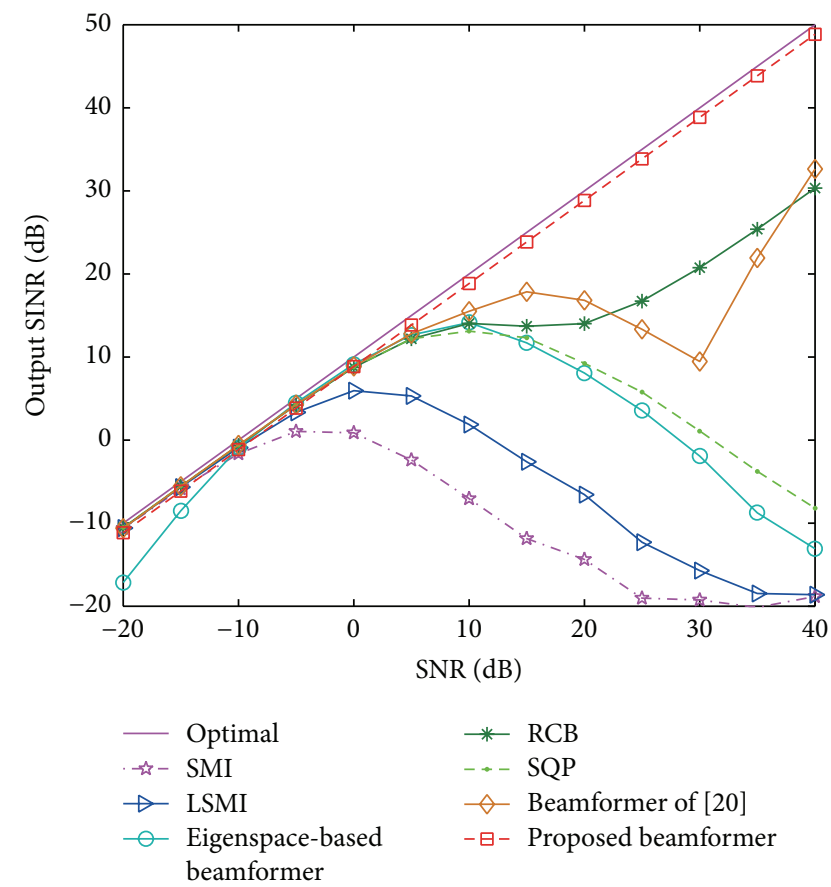

FIGURE 5: Output SINR of beamformers versus input SNR.

in each simulation run. In this paper, we set $T=4 . \theta_{i}$ comes from a uniform random generator with mean $3^{\circ}$ and standard deviation $1^{\circ}$. Other simulation parameters are the same as Simulation Example 3.

The normalized beampatterns of the beamformers are shown in Figure 6. It is observed that SMI completely fails in a coherent local scattering environment, which produces a null in the desired signal. Additionally, the side lobe level of SMI is significantly increased. The performance of RCB is better than SMI. The proposed method has best performance for interference suppression, which provides deep and wide nulls in inference directions. Furthermore, its response peaks are located at the actual direction.

Figure 7 shows the output SINR of the beamformers versus SNR with $K=200$. We can see that the output SINR of the SMI sharply decreased with an increment of SNR. The proposed algorithm enjoys highest SINR compared with commonly used beamformers.

Simulation Example 5 (evaluation on both the ASV mismatch and jammer nonstationarity). The influence of both ASV mismatch and moving jammer on the array output SINR is presented and discussed in detail. The desired signal direction is still set at $0^{\circ}$, whereas the presumed one is set at $3^{\circ}$. The directions of the moving interferences are time-varying which are described as $\theta(k)=-45^{\circ}+0.01^{\circ} i$ and $\theta(k)=-45^{\circ}+$ $0.04^{\circ} i$, respectively. In this example, the diagonal loading Mailloux beamforming (DL-Mailloux) is considered, and the diagonal loading factor is selected as twice as the noise power.

Figures 8-9 show the output SINR in the presence of the ASV mismatch and moving jammer. The proposed method 


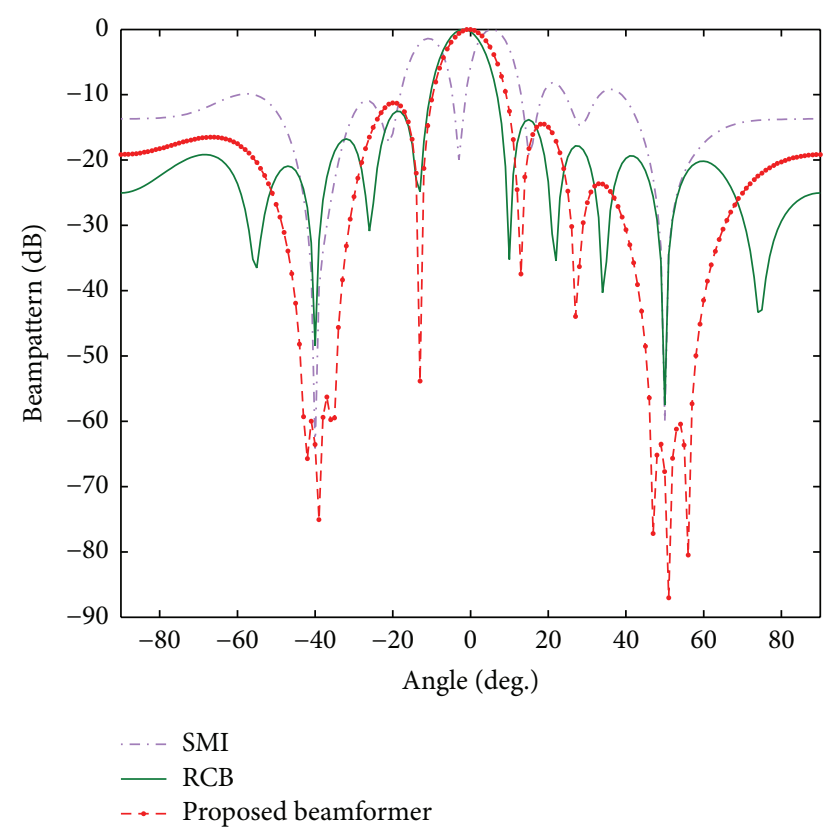

FIGURE 6: Normalized beampattern plots in absence of ASV mismatch due to coherent local scattering.

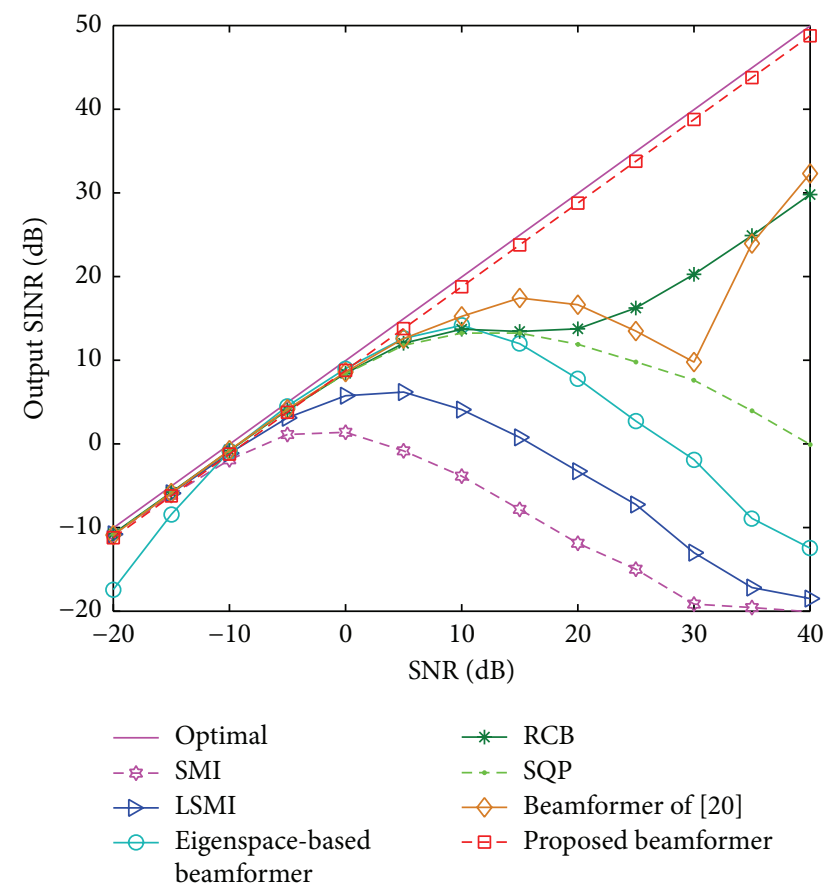

FIGURE 7: Output SINR of beamformers versus input SNR.

works well in the presence of these errors, which consistently enjoys the best performance due to its strong ability to reduce the systematic error. The output SINR of the SMI decreases sharply with increased SNR. It is worthwhile to note that the performance of DL-Mailloux's method is better than Mailloux's method for it combines the advantages of both diagonal loading and Mailloux's methods. We also can find

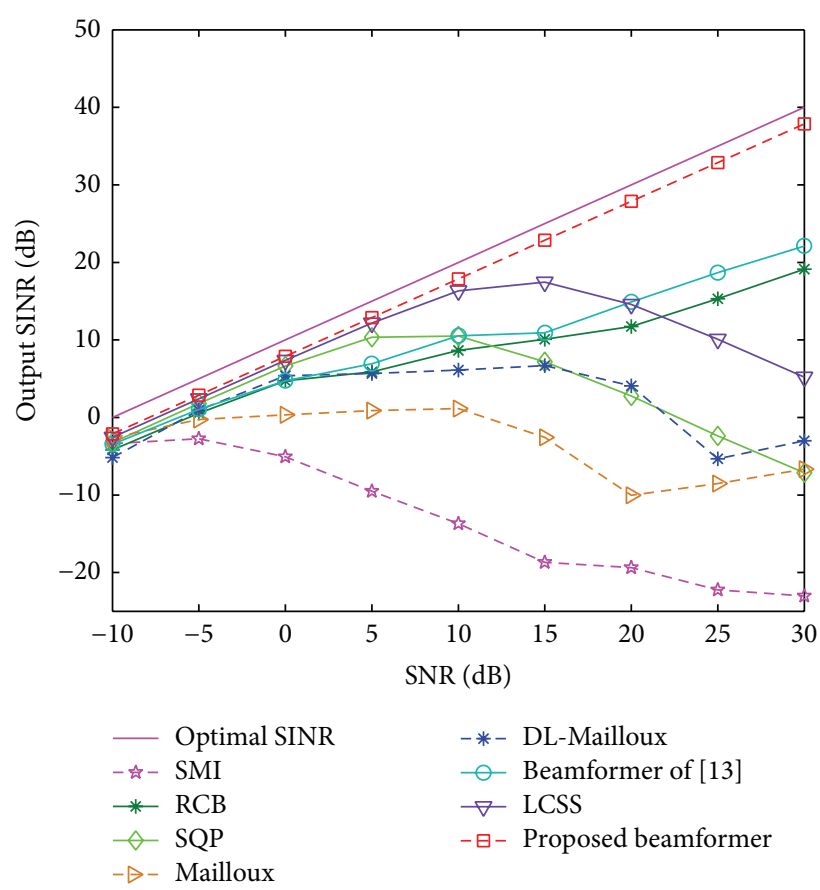

FIGURE 8: Output SINR of beamformers versus input $\operatorname{SNR}\left(v_{\theta}=\right.$ $0.01^{\circ}$ per snapshot).

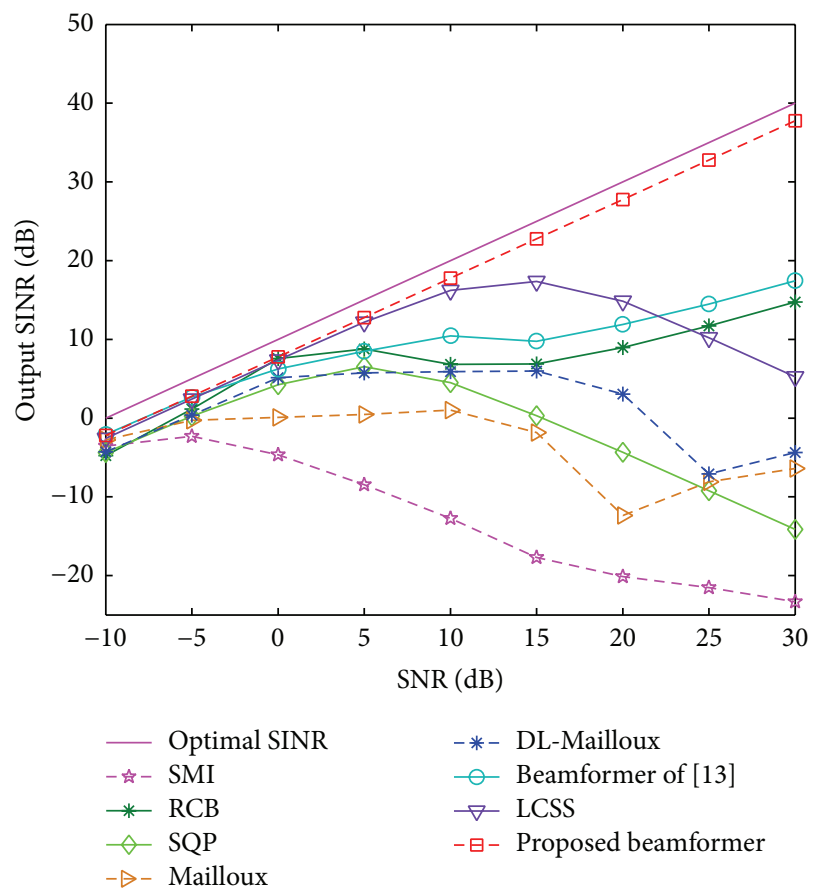

FIGURE 9: Output SINR of beamformers versus input SNR $\left(v_{\theta}=\right.$ $0.04^{\circ}$ per snapshot).

that the performance of the Mailloux method, RCB, and SQP obviously degrades with the increment of $v_{\theta}$. The proposed beamformer is insensitive to $v_{\theta}$, which always obtain a high SINR that is very close to the optimal one. 
TABle 1: Performance comparison of tested beamformers.

\begin{tabular}{|c|c|c|c|}
\hline \multirow[b]{2}{*}{ Beamformers } & \multirow[b]{2}{*}{ Input SNR (dB) } & \multicolumn{2}{|c|}{ Output SINR (dB) } \\
\hline & & $\begin{array}{c}v_{\theta}=0.01^{\circ} \text { per snapshot, } \\
3^{\circ} \text { steering direction } \\
\text { error }\end{array}$ & $\begin{array}{c}v_{\theta}=0.04^{\circ} \text { per snapshot } \\
3^{\circ} \text { steering direction } \\
\text { error }\end{array}$ \\
\hline SMI-MVDR & 10 & -13.696 & -12.732 \\
\hline $\mathrm{RCB}$ & 10 & 8.655 & 6.832 \\
\hline Mailloux & 10 & 1.455 & 1.014 \\
\hline DL-Mailloux & 10 & 6.608 & 6.201 \\
\hline SQP & 10 & 10.418 & 4.490 \\
\hline Beamformer of [20] & 10 & 9.085 & 7.049 \\
\hline Beamformer of [13] & 10 & 10.414 & 10.268 \\
\hline LCSS & 10 & 16.321 & 16.226 \\
\hline Proposed beamformer & 10 & 17.852 & 17.663 \\
\hline
\end{tabular}

In order to further compare the performance of beamformers, output SINRs of the different beamformers for input SNR is $10 \mathrm{~dB}$ are discussed in Table 1.

It can be seen from Table 1 that the proposed beamformer has obvious advantages over the SQP, beamformer of [20], and beamformer of [13], which has the highest SINR in different $v_{\theta}$.

\section{Impacts of Some Factors on Performance}

The goal of this section is to investigate the impacts of parameters $M$ and $\lambda$ on performance.

Simulation Example 6. In this simulation, the actual desired signal direction is $0^{\circ}$, and the presumed one is also at $0^{\circ}$. We set the width of the null as $10^{\circ}$, the diagonal loading factor $\lambda=$ 0.0100 , whereas the number of the base vectors $M$ is varied. The normalized beampatterns with varying $M$ are shown in Figure 10, and the output SINR is displayed in Figure 11.

It can be seen from Figure 10 that $M$ has an important effect on the performance of the proposed method. The null depth is in proportion to $M$, and the main lobe becomes wider with the increase of $M$. When $M$ is too small, the null may not be deep enough to suppress the interferences. The broadened main lobe can improve the convergence speed when the system suffers from high steering errors [26], but when $M$ is too large, for example, $M=8$, we can find from Figure 10 that the main lobe is distorted. An effective solution for finding a proper value of $M$ given a desired null width and depth is very important for the proposed method.

We assume the nominal direction of interference is $\widehat{\theta}_{p}$, the desired null width is $\delta$, and depth is $\eta$. The output power at the trough is

$$
P_{\text {out }}=\widetilde{\mathbf{W}}^{H} \mathbf{Q} \widetilde{\mathbf{W}},
$$

where $\mathbf{Q}=\int_{\theta_{p}-\delta / 2}^{\theta_{p}+\delta / 2} \overline{\mathbf{a}}(\theta) \overline{\mathbf{a}}^{H}(\theta) d \theta$ and $\widetilde{\mathbf{W}}$ is the adaptive weight vector. For a given null depth $\eta$, it is equal to the following implementation:

$$
P_{\text {out }}=\widetilde{\mathbf{W}}^{H} \mathbf{Q} \widetilde{\mathbf{W}} \leq \eta \text {. }
$$

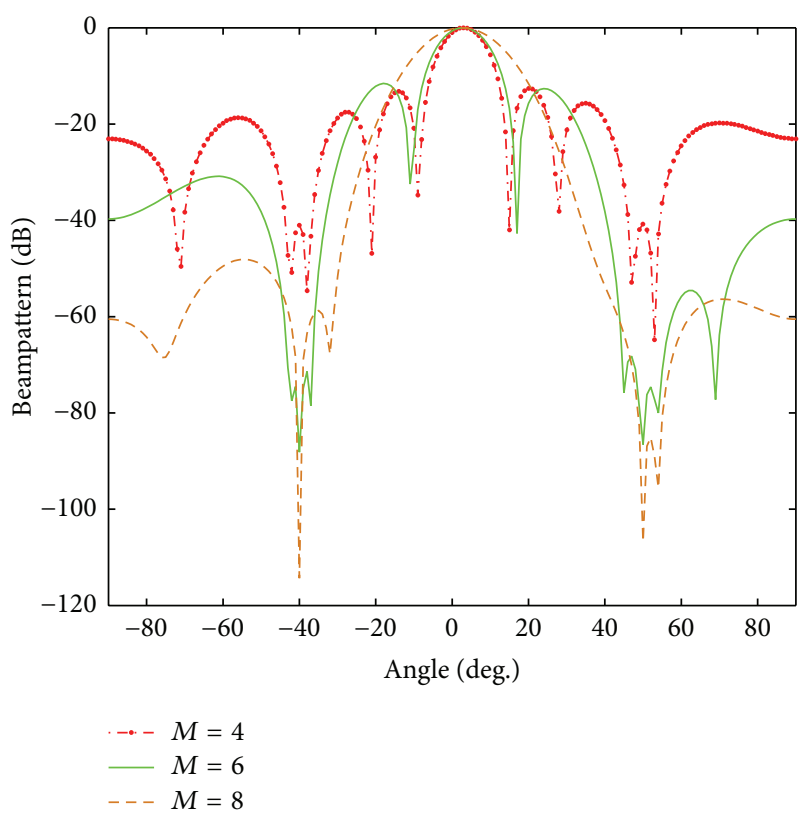

FIGURE 10: Normalized beampattern plots.

Since the null depth is in proportion to $M$ as the simulation results demonstrated in Figure 10, our responsibility is to find smallest $M$ under constraint (34). The problem can be solved as the following steps:

(1) Initialization, $M=1$.

(2) Update the projection matrix $\mathbf{T}$ as $\mathbf{T}=\sum_{n=1}^{M} \overline{\boldsymbol{v}}_{\theta_{n}} \overline{\boldsymbol{v}}_{\theta_{n}}^{H}$.

(3) Calculate the adaptive weight vector $\widetilde{\mathbf{W}}$ using (29).

(4) Calculate the output power at the trough $P_{\text {out }}=$ $\widetilde{\mathbf{W}}^{H} \mathbf{Q} \widetilde{\mathbf{W}}$.

(5) If $P_{\text {out }} \leq \eta$, end; else, $M=M+1$, go to step (2).

In order to demonstrate the effectiveness of the above method, some simulation experiments have been given as follows. 


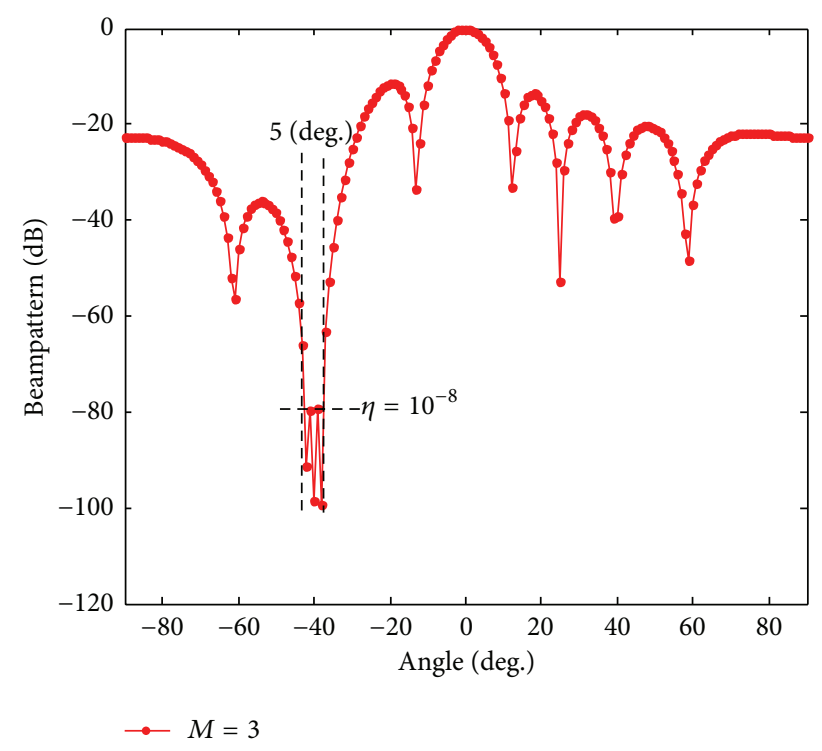

FIGURE 11: Normalized beampatterns for $\delta=5^{\circ}$ and $\eta=10^{-8}$.

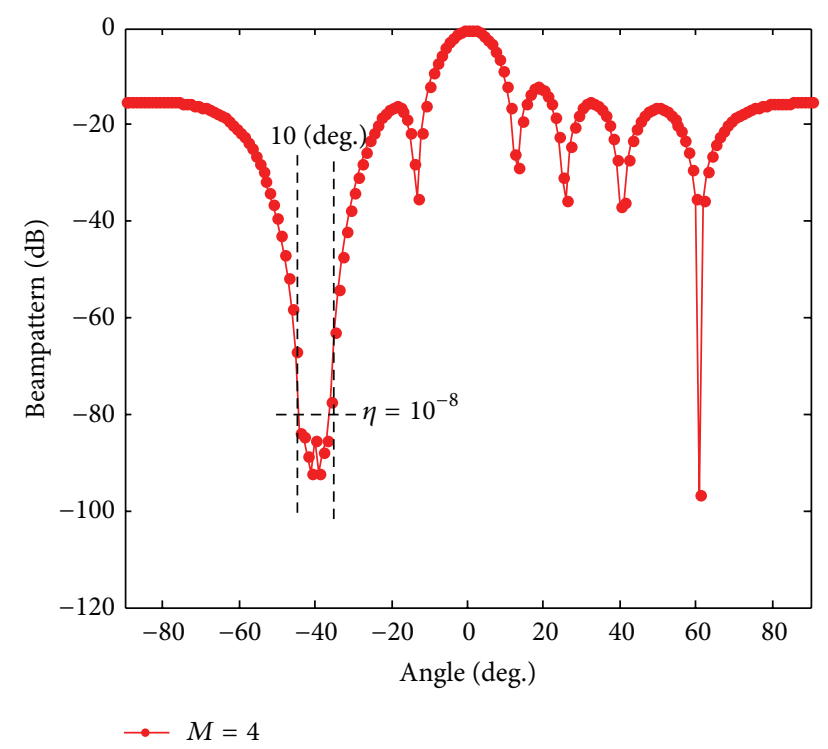

FIGURE 12: Normalized beampatterns for $\delta=10^{\circ}$ and $\eta=10^{-8}$.

Simulation Example 7. In this simulation, the actual desired signal direction is $0^{\circ}$, and the presumed one is also $0^{\circ}$. The input SNR is $0 \mathrm{~dB}$. We set the widths of the null as $5^{\circ}$ and $10^{\circ}$, respectively. The predefined null depths are $\eta=10^{-8}$ and $\eta=10^{-5}$, respectively. The diagonal loading factor $\lambda=$ 0.0100 . An interference is from the direction $-40^{\circ}$ with INR $=30 \mathrm{~dB}$. The number of snapshots is $K=100$. The normalized beampatterns are shown in Figures 11-14.

It can be seen from these figures that, by giving a desired null width $\delta$ and a null depth $\eta$, the proper value of $M$ can be obtained by using the above method. It is also found that the smaller null depth $\eta$ is, the larger $M$ is needed under a certain null width $\delta$. The actual null depth may be deeper than

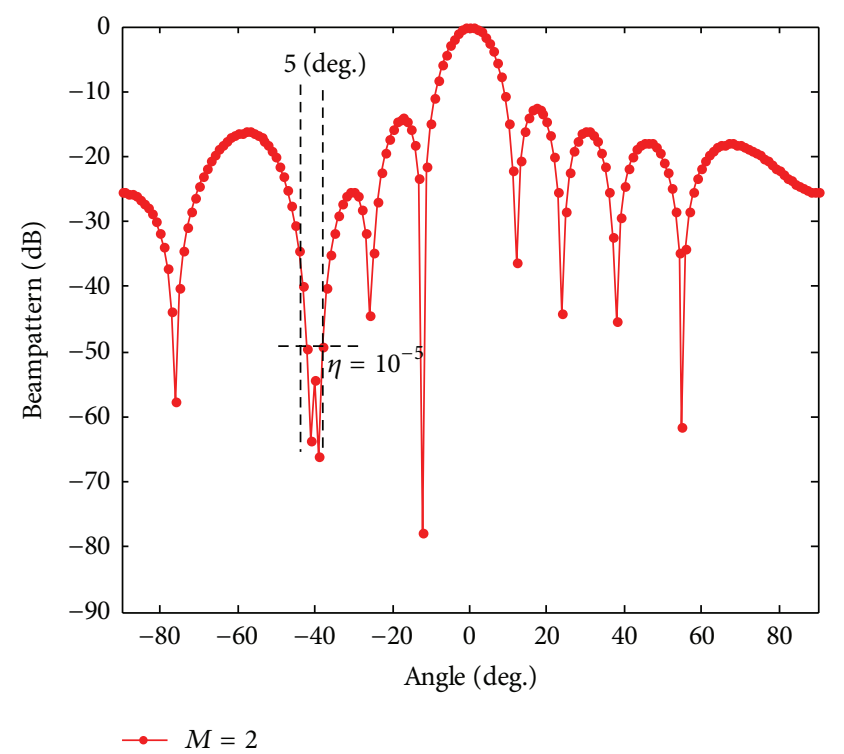

FIGURE 13: Normalized beampatterns for $\delta=5^{\circ}$ and $\eta=10^{-5}$.

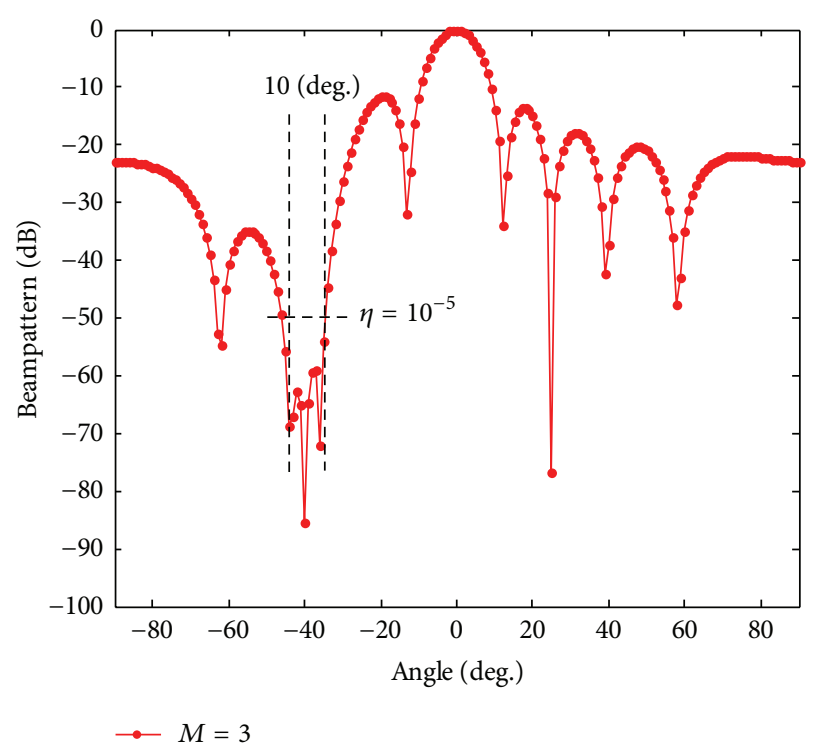

FIGURE 14: Normalized beampatterns for $\delta=10^{\circ}$ and $\eta=10^{-5}$.

the desired one as $M$ is an integer. We need to point out that $1 \leq M \leq N$ as shown in (13). We can see from Figure 10 that the main lobe of the beampattern is distorted when $M=8$. Thus, the null depth $\eta$ should be larger than $10^{-10}$.

Simulation Example 8. In this experiment, the number of the base vectors is $M=6$, whereas diagonal loading factor $\lambda$ is varying. The actual desired signal direction is $0^{\circ}$, and the presumed one is $3^{\circ}$. The input SNR is $0 \mathrm{~dB}$. We set the width of the null as $10^{\circ}$. Two independent interferences are from the directions $-40^{\circ}$ and $50^{\circ}$ with INR $=30 \mathrm{~dB}$. The number of snapshots is $K=100$. 
TABLE 2: Output SINRs versus $\lambda$ with different ASV errors (input SNR at $10 \mathrm{~dB}$ ).

\begin{tabular}{|c|c|c|c|c|c|c|c|c|c|c|}
\hline \multirow{3}{*}{ Angle error } & \multirow{3}{*}{ Optimal SINR (dB) } & \multicolumn{9}{|c|}{ Output SINR (dB) } \\
\hline & & \multicolumn{5}{|c|}{ Proposed beamformer } & \multirow{2}{*}{$\mathrm{RCB}$} & \multirow{2}{*}{ SQP } & \multirow{2}{*}{ Method of [20] } & \multirow{2}{*}{ LCSS } \\
\hline & & $\lambda=0.0000$ & $\lambda=0.1000$ & $\lambda=0.0100$ & $\lambda=0.0010$ & $\lambda=0.0001$ & & & & \\
\hline $0^{\circ}$ & 19.9629 & 12.6189 & 19.5183 & 19.5102 & 19.5096 & 19.5093 & 13.9363 & 16.0907 & 15.6839 & 17.5234 \\
\hline $1^{\circ}$ & 19.9629 & 12.2875 & 19.4344 & 19.4261 & 19.4257 & 19.4256 & 12.2046 & 15.2841 & 15.3738 & 17.4056 \\
\hline $2^{\circ}$ & 19.9629 & 14.0490 & 19.1642 & 19.1567 & 19.1563 & 19.1562 & 10.8935 & 13.5607 & 15.7175 & 16.9827 \\
\hline $3^{\circ}$ & 19.9629 & 13.1785 & 18.6815 & 18.6762 & 18.6757 & 18.6755 & 9.9799 & 11.0069 & 15.0569 & 16.8593 \\
\hline $4^{\circ}$ & 19.9629 & 13.1317 & 17.965 & 17.9621 & 17.9618 & 17.9616 & 9.8641 & 8.1054 & 15.2996 & 16.2682 \\
\hline $5^{\circ}$ & 19.9629 & 13.8695 & 16.9978 & 16.9964 & 16.9959 & 16.9958 & 11.8140 & 5.7440 & 15.2911 & 15.7629 \\
\hline $6^{\circ}$ & 19.9629 & 13.3528 & 15.762 & 15.9637 & 15.9634 & 15.9634 & 11.0708 & 3.3879 & 14.9117 & 15.1536 \\
\hline
\end{tabular}

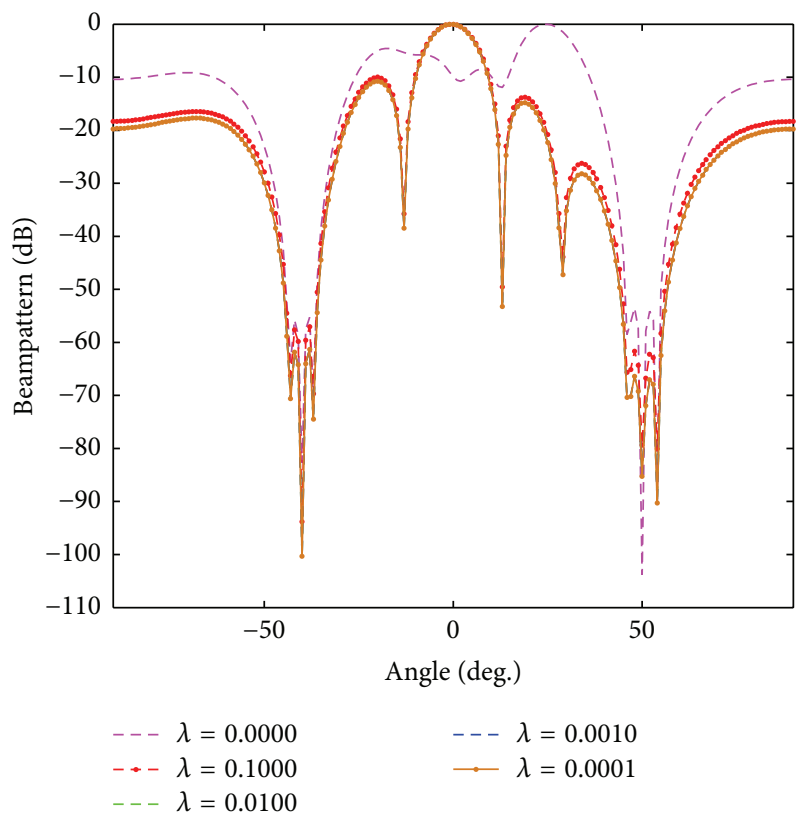

FIGURE 15: Normalized beampatterns.

The normalized beampatterns for $\lambda=0.0000, \lambda=0.1000$, $\lambda=0.0100, \lambda=0.0010$, and $\lambda=0.0001$ are shown in Figure 15, while the output SINRs versus $\lambda$ are displayed in Figure 16. It is observed that the diagonal loading factor is very important to the proposed beamformer: when $\lambda=$ 0.0000 , the beam pattern is seriously distorted and the output SINRs degrade seriously. But, luckily, the performance of the proposed beamformer is not sensitive to the parameter $\lambda$, the beampatterns are almost invariable, and the performance difference is very small when $\lambda \neq 0.0000$.

In order to further study the impacts of $\lambda$ on performance, the output SINRs versus $\lambda$ with different ASV errors are shown in Table 2 . It can be seen that the output SINR differences for $\lambda=0.0100, \lambda=0.0010$, and $\lambda=$ 0.0001 are very small with a particular ASV error, which indicates that the proposed beamformer can provide a good performance over a wide range of $\lambda$. Though the output SINR of the proposed method degraded with the increase of ASV error, it is still prior to other tested beamformers,

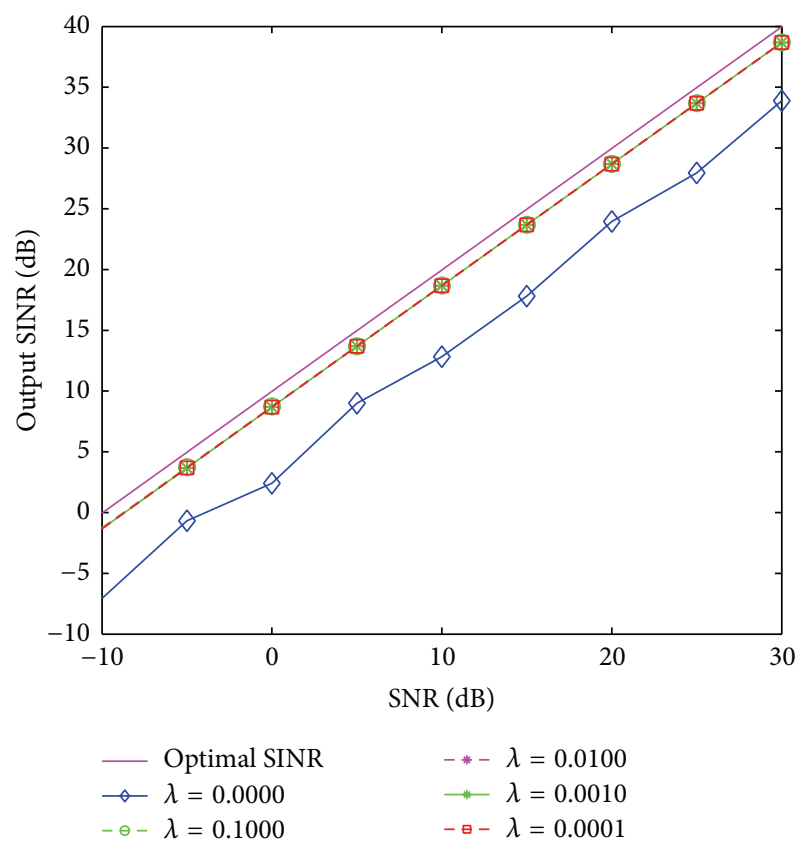

FIGURE 16: Output SINR versus $\lambda$ with $3^{\circ}$ steering direction error.

which indicates the proposed beamformer can provide a good performance over a wide range of $\lambda$ in the presence of different ASV errors. Thus, our proposed method can provide better operability and practicality than those of the previously reported methods. Additionally, $0.0100 \leq \lambda \leq 0.0001$ is suggested for the proposed algorithm.

\section{Conclusions}

A novel robust null broadening beamforming against ASV mismatch and jammer motion has been proposed and extensively discussed in this paper. With a new projection matrix constructed, the proposed beamformer was realized by the combination of projection transform and diagonal loading techniques. The projection matrix plays a role in expanding the incident directions of interferences and improving the orthogonality between the signal-plus-interference and noise subspaces; thus the wide and deep null can be obtained in 
the adapted pattern. The proposed beamformer can provide strong robustness against not only fast-moving interferer but also ASV mismatch. The detailed theoretical analysis and numerical examples were presented to discuss the advantages of the proposed beamformer. The simulation results demonstrated that the proposed method can achieve a better performance than other existing methods.

\section{Conflict of Interests}

The authors declare that there is no conflict of interests regarding the publication of this paper.

\section{Acknowledgments}

This paper was supported by National Defense "973" Basic Research Development Program of China (no. 6131380101). This paper is also supported by Pre-Research Fund of the 12th Five-Year Plan (no. 4010403020102) and the Fundamental Research Funds for the Central Universities (HEUCFD1433, HEUCF1508).

\section{References}

[1] H. L. van Trees, Optimum Array Processing, Wiley, New York, NY, USA, 2002.

[2] H. Li, K. Wang, C. Wang, Y. He, and X. Zhu, "Robust adaptive beamforming based on worst-case and norm constraint," International Journal of Antennas and Propagation, vol. 2015, Article ID 765385, 7 pages, 2015.

[3] H. Singh and R. M. Jha, "Trends in adaptive array processing," International Journal of Antennas and Propagation, vol. 2012, Article ID 361768, 20 pages, 2012.

[4] S. A. Vorobyov, A. B. Gershman, and Z.-Q. Luo, "Robust adaptive beamforming using worst-case performance optimization: a solution to the signal mismatch problem," IEEE Transactions on Signal Processing, vol. 51, no. 2, pp. 313-324, 2003.

[5] J. Li, P. Stoica, and Z. Wang, "Doubly constrained robust Capon beamformer," IEEE Transactions on Signal Processing, vol. 52, no. 9, pp. 2407-2423, 2004.

[6] H. Song, W. A. Kuperman, W. S. Hodgkiss, P. Gerstoft, and J. S. Kim, "Null broadening with snapshot-deficient covariance matrices in passive sonar," IEEE Journal of Oceanic Engineering, vol. 28, no. 2, pp. 250-261, 2003.

[7] R. J. Mailloux, "Covariance matrix augmentation to produce adaptive array pattern troughs," Electronics Letters, vol. 31, no. 10, pp. 771-772, 1995.

[8] M. Zatman, "Production of adaptive array troughs by dispersion synthesis," Electronics Letters, vol. 31, no. 25, pp. 2141-2142, 1995.

[9] J. R. Guerci, "Theory and application of covariance matrix tapers for robust adaptive beamforming," IEEE Transactions on Signal Processing, vol. 47, no. 4, pp. 977-985, 1999.

[10] A. B. Gershman, U. Nickel, and J. F. Böhme, "Adaptive beamforming algorithms with robustness against jammer motion," IEEE Transactions on Signal Processing, vol. 45, no. 7, pp. 1878$1885,1997$.

[11] A. B. Gershman, G. V. Serebryakov, and J. F. Böhme, "Constrained hung-turner adaptive beam-forming algorithm with additional robustness to wideband and moving jammers," IEEE
Transactions on Antennas and Propagation, vol. 44, no. 3, pp. 361-367, 1996.

[12] M. Zatman, "Comments on 'theory and application of covariance matrix tapers for robust adaptive beamforming"' IEEE Transactions on Signal Processing, vol. 48, no. 6, pp. 1796-1800, 2000.

[13] S. A. Vorobyov, A. B. Gershman, Z.-Q. Luo, and N. Ma, "Adaptive beamforming with joint robustness against mismatched signal steering vector and interference nonstationarity," IEEE Signal Processing Letters, vol. 11, no. 2, pp. 108-111, 2004.

[14] A. Amar and M. A. Doron, "A linearly constrained minimum variance beamformer with a pre-specified suppression level over a pre-defined broad null sector," Signal Processing, vol. 109, pp. 165-171, 2015.

[15] B. D. Carlson, "Covariance matrix estimation errors and diagonal loading in adaptive arrays," IEEE Transactions on Aerospace and Electronic Systems, vol. 24, no. 4, pp. 397-401, 1988.

[16] A. Elnashar, S. M. Elnoubi, and H. A. El-Mikati, "Further study on robust adaptive beamforming with optimum diagonal loading," IEEE Transactions on Antennas and Propagation, vol. 54, no. 12, pp. 3647-3658, 2006.

[17] L. Du, J. Li, and P. Stoica, "Fully automatic computation of diagonal loading levels for robust adaptive beamforming," IEEE Transactions on Aerospace and Electronic Systems, vol. 46, no. 1, pp. 449-458, 2010.

[18] J. Li, P. Stoica, and Z. Wang, "On robust Capon beamforming and diagonal loading," IEEE Transactions on Signal Processing, vol. 51, no. 7, pp. 1702-1715, 2003.

[19] A. Hassanien, S. A. Vorobyov, and K. M. Wong, "Robust adaptive beamforming using sequential quadratic programming: an iterative solution to the mismatch problem," IEEE Signal Processing Letters, vol. 15, pp. 733-736, 2008.

[20] A. Khabbazibasmenj, S. A. Vorobyov, and A. Hassanien, "Robust adaptive beamforming based on steering vector estimation with as little as possible prior information," IEEE Transactions on Signal Processing, vol. 60, no. 6, pp. 2974-2987, 2012.

[21] S. A. Vorobyov, "Principles of minimum variance robust adaptive beamforming design," Signal Processing, vol. 93, no. 12, pp. 3264-3277, 2013.

[22] L. Chang and C.-C. Yeh, "Performance of DMI and eigenspacebased beamformers," IEEE Transactions on Antennas and Propagation, vol. 40, no. 11, pp. 1336-1347, 1992.

[23] D. D. Feldman and L. J. Griffiths, "Projection approach for robust adaptive beamforming," IEEE Transactions on Signal Processing, vol. 42, no. 4, pp. 867-876, 1994.

[24] P. Stoica and R. L. Moses, Introduction to Spectral Analysis, Prentice-Hall, Englewood Cliffs, NJ, USA, 1997.

[25] R. Schmidt, "Multiple emitter location and signal parameter estimation," IEEE Transactions on Antennas and Propagation, vol. 34, no. 3, pp. 276-280, 1986.

[26] Z. Xu and Y. Zakharov, "Modified null broadening adaptive beamforming: constrained optimisation approach," Electronics Letters, vol. 43, no. 3, pp. 145-146, 2007. 

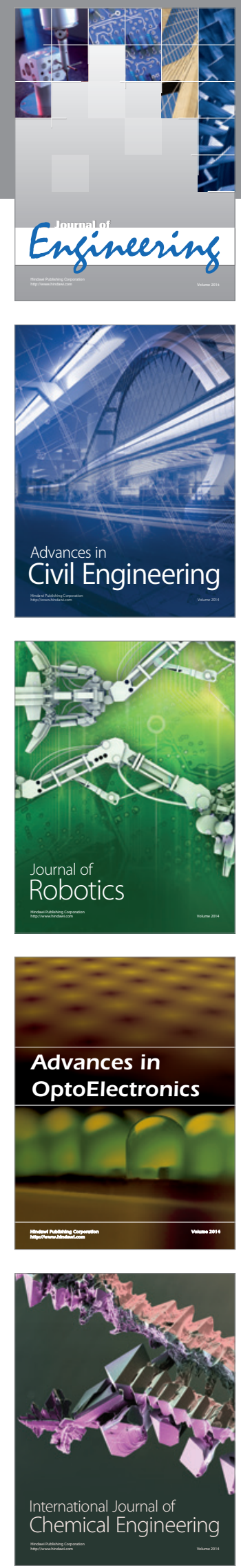

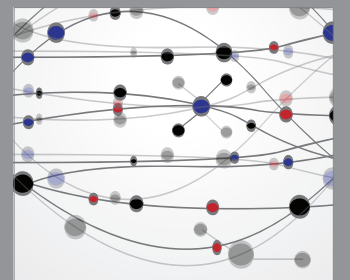

The Scientific World Journal
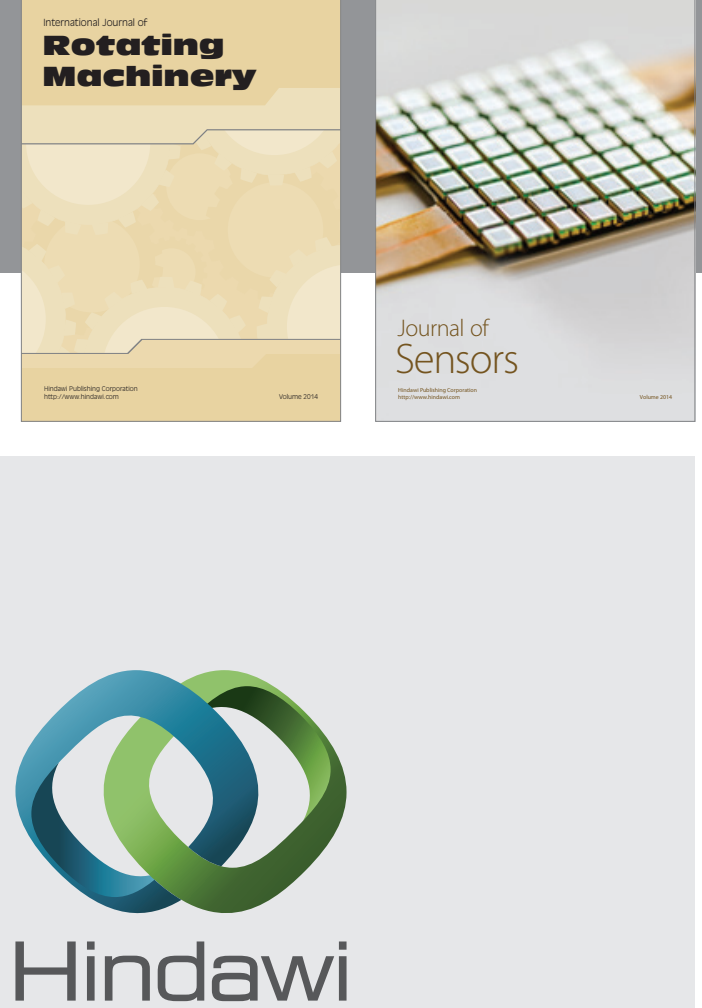

Submit your manuscripts at http://www.hindawi.com
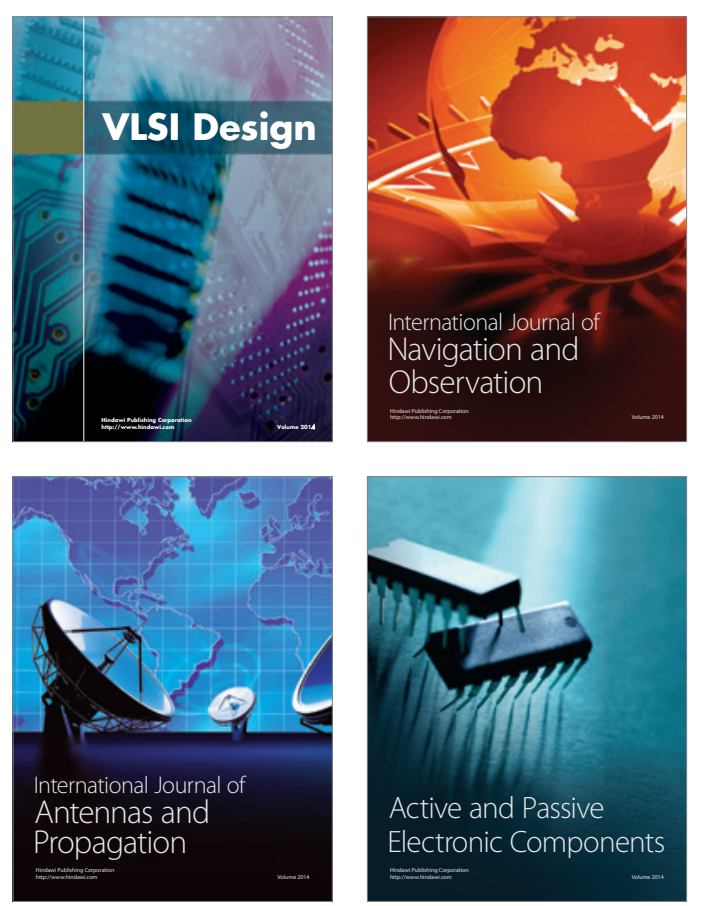
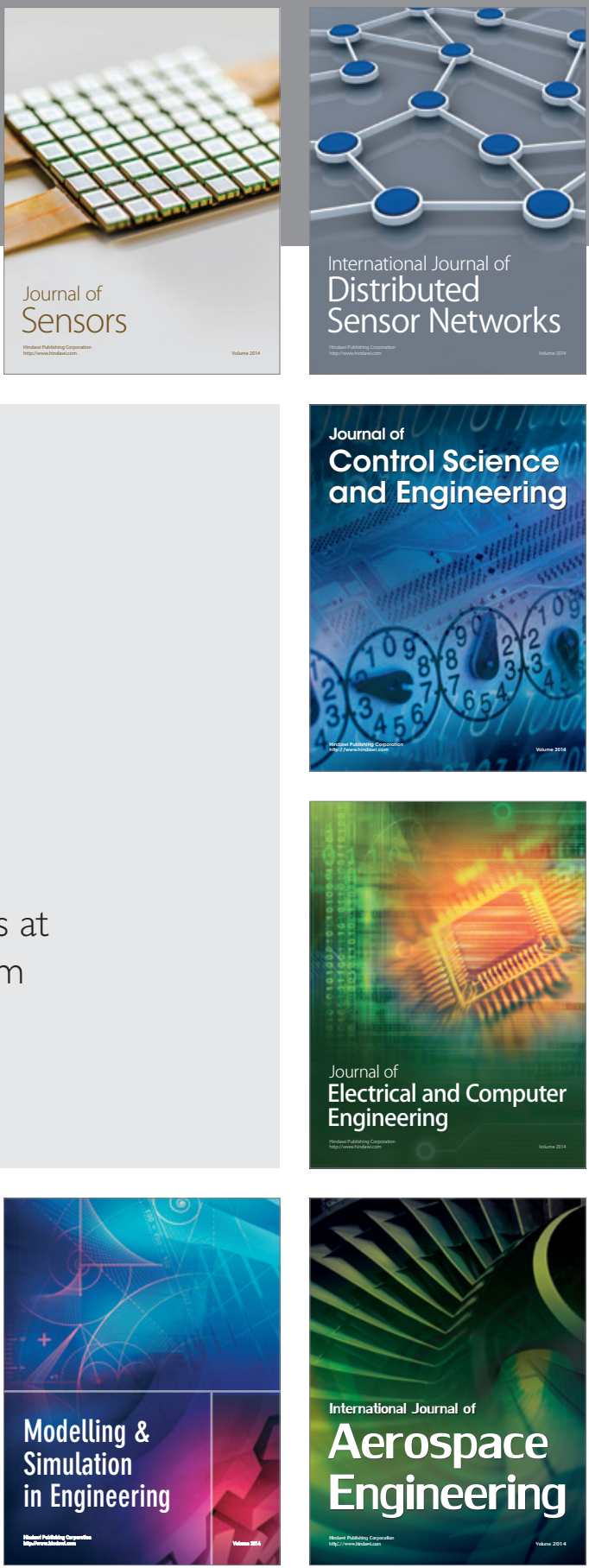

Journal of

Control Science

and Engineering
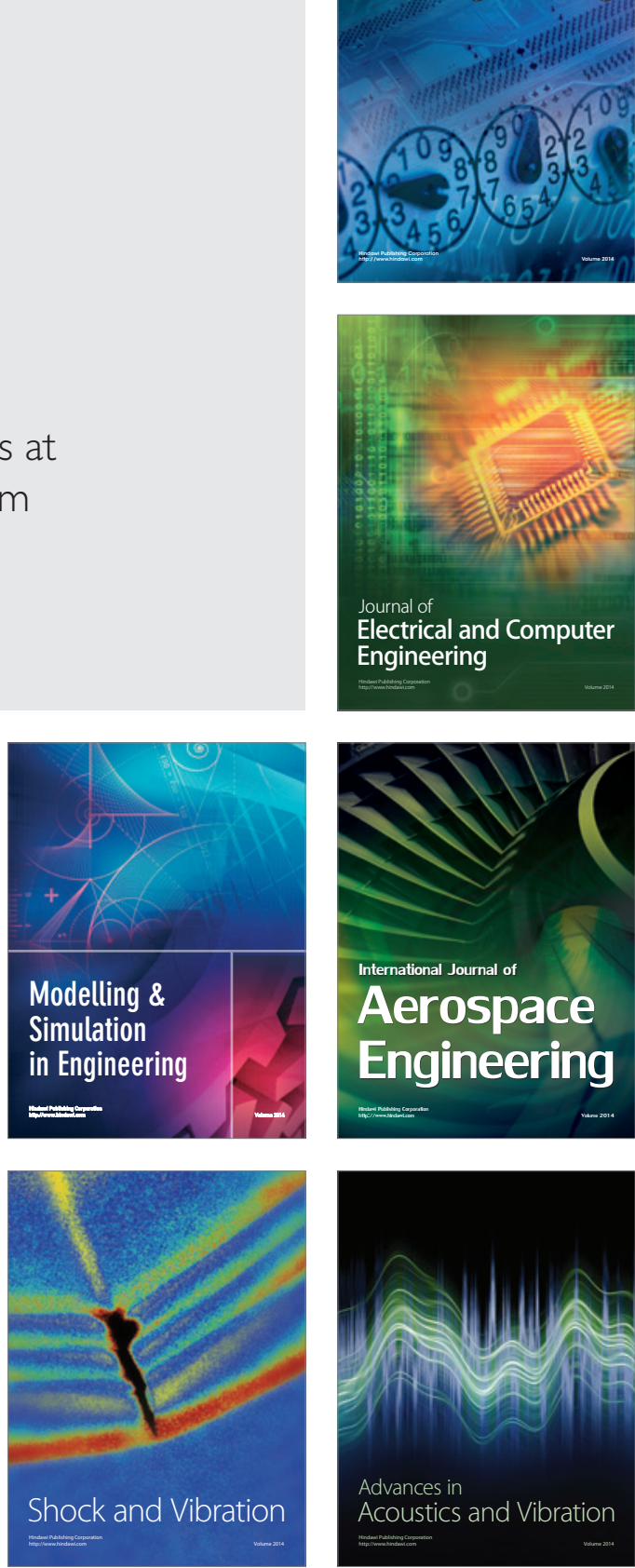